\title{
A combined prediction model for biliary tract cancer using the prognostic nutritional index and pathological findings: a single-center retrospective study
}

Masashi Utsumi* Koji Kitada, Naoyuki Tokunaga, Takamitsu Kato, Toru Narusaka, Ryosuke Hamano, Hideaki Miyasou, Yousuke Tsunemitsu, Shinya Otsuka and Masaru Inagaki

\begin{abstract}
Background: The prognostic nutritional index, a marker of nutritional status and systemic inflammation, is a known biomarker for various cancers. However, few studies have evaluated the predictive value of the prognostic nutritional index in patients with biliary tract cancer. Therefore, we investigated the prognostic significance of the prognostic nutritional index, and developed a risk-stratification system to identify prognostic factors in patients with biliary tract cancer.

Methods: Between July 2010 and March 2021, 117 patients with biliary tract cancer were recruited to this singlecenter, retrospective study. The relationship between clinicopathological variables, including the prognostic nutritional index, and overall survival was analyzed using univariate and multivariate analyses. A $P<0.05$ was considered statistically significant.

Results: The median age was 75 (range 38-92) years. Thirty patients had intrahepatic cholangiocarcinoma; 29, gallbladder carcinoma; 27, distal cholangiocarcinoma; 17, ampullary carcinoma; and 13, perihilar cholangiocarcinoma. Curative (RO) resection was achieved in 99 patients. In univariate analysis, the prognostic nutritional index $(<42)$, lymph node metastasis, carbohydrate antigen $19-9$ level $(>20 \mathrm{U} / \mathrm{mL}$ ), preoperative cholangitis, tumor differentiation, operation time ( $\geq 360 \mathrm{~min})$, and $\mathrm{R} 1-2$ resection were significant risk factors for overall survival. The prognostic nutritional index $(P=0.027)$, lymph node metastasis $(P=0.040)$, and tumor differentiation $(P=0.006)$ were independent prognostic factors in multivariate analysis. A combined score of the prognostic nutritional index and pathological findings outperformed each marker alone, in terms of discriminatory power.

Conclusions: The prognostic nutritional index, lymph node metastasis, and tumor differentiation were independent prognostic factors after surgical resection in patients with biliary tract cancer. A combined prediction model using the prognostic nutritional index and pathological findings accurately predicted prognosis, and can be used as a novel prognostic factor in patients with biliary tract cancer.
\end{abstract}

Keywords: Biliary tract cancer, Inflammation-based prognostic score, Prognosis, Prognostic nutritional index, Surgical resection

\footnotetext{
${ }^{*}$ Correspondence: masasi11232001@yahoo.co.jp

Department of Surgery, National Hospital Organization Fukuyama

Medical Center, 4-14-17 Okinogami-cho, Fukuyama City, Hiroshima

720-8520, Japan
}

(C) The Author(s) 2021. Open Access This article is licensed under a Creative Commons Attribution 4.0 International License, which permits use, sharing, adaptation, distribution and reproduction in any medium or format, as long as you give appropriate credit to the original author(s) and the source, provide a link to the Creative Commons licence, and indicate if changes were made. The images or other third party material in this article are included in the article's Creative Commons licence, unless indicated otherwise in a credit line to the material. If material is not included in the article's Creative Commons licence and your intended use is not permitted by statutory regulation or exceeds the permitted use, you will need to obtain permission directly from the copyright holder. To view a copy of this licence, visit http://creativecommons.org/licenses/by/4.0/. The Creative Commons Public Domain Dedication waiver (http://creativeco mmons.org/publicdomain/zero/1.0/) applies to the data made available in this article, unless otherwise stated in a credit line to the data. 


\section{Background}

Biliary tract cancer (BTC), including gallbladder carcinoma (GBC), cholangiocarcinoma, and ampullary carcinoma, is a relatively rare, but aggressive malignancy [1]. Despite its rarity, the incidence of BTC has steadily increased in recent decades [1]. Radical resection is the only curative treatment option for BTC. However, the high recurrence rate is a major concern [2]. Moreover, BTC is usually diagnosed at an advanced stage, at which point most patients cannot be considered as candidates for radical resection. Despite recent developments in surgical techniques and adjuvant chemotherapy, the prognosis remains poor [3, 4]. Preoperative prognostic factors for BTC may allow better risk-benefit assessment before surgery, and permit patient stratification for more personalized treatment [5]. Therefore, it is essential to identify new predictive biomarkers.

The prognostic nutritional index (PNI) is a marker of nutritional status and systemic inflammation, based on serum albumin concentration and total lymphocyte count, both of which can be easily obtained from routine preoperative blood tests [6]. Several studies [7-11] have shown that the PNI can be used as a prognostic marker in patients with various cancers. However, few studies have evaluated the prognostic value of the PNI in patients with BTC. Therefore, in this single-center, retrospective study, we investigated the prognostic significance of the PNI in patients with BTC, and explored its potential clinical application. We also compared the PNI to other inflammation-based prognostic scores, including the Glasgow Prognostic Score (GPS) [12], C-reactive protein (CRP) to albumin ratio (CAR) [13], neutrophil to lymphocyte ratio (NLR), and platelet to lymphocyte ratio (PLR) [14, 15]. Furthermore, we developed a risk-stratification system combining clinicopathological predictors to identify prognostic factors in patients with BTC.

\section{Methods}

\section{Patients}

A total of 117 consecutive patients who underwent surgical resection for BTC at the Department of Surgery, National Hospital Organization Fukuyama Medical Center, Hiroshima, Japan, between July 2010 and March 2021 were retrospectively reviewed. BTC included GBC, intrahepatic cholangiocarcinoma (ICC), distal cholangiocarcinoma, ampullary carcinoma, and perihilar cholangiocarcinoma, as confirmed by imaging and pathological examination. One patient died of heart failure due to arrhythmia on postoperative day 18 during the perioperative period. This patient was excluded from the study. Consequently, a total of 116 patients who underwent surgical resection for BTC were analyzed.

\section{Data collection}

Clinicopathological data were obtained retrospectively from patients' medical records, including demographic information (age at surgery and sex), laboratory data (CRP level, serum albumin concentration, platelet count, neutrophil count, lymphocyte count, and tumor markers), comorbidities (hypertension, diabetes mellitus, cardiac disease, and stroke), preoperative cholangitis, operative procedure (i.e., type of resection), operative blood loss, operation time, transfusion, tumor stage (Union for International Cancer Control TumorNode-Metastasis [TNM] classification [sixth edition]) [16], tumor differentiation, and postoperative adjuvant chemotherapy. The extent of hepatic resection was classified according to the Brisbane 2000 nomenclature [17]. Major hepatectomy was defined as the resection of $\geq 3$ contiguous liver segments, according to Couinaud's classification [18], while minor hepatectomy was defined as the resection of $<3$ contiguous liver segments, or nonanatomic partial resection. Curative (R0) resection was defined as complete removal of all macroscopic nodules with microscopically clear margins. R1 and R2 resections were defined as microscopic or macroscopic disease, respectively, involving $\geq 1$ margin. Complications were defined according to the Clavien-Dindo classification [19]. In this study, postoperative complications were defined as complications of Clavien-Dindo grade $\geq$ IIIa. Postoperative mortality was defined as death from any cause within 30 days after surgery.

\section{PNI and other inflammation-based prognostic scores}

Peripheral venous blood samples were collected within 2 weeks before surgery. The PNI was calculated as $10 \times$ serum albumin concentration $(\mathrm{g} / \mathrm{dL})+0.05 \times$ total lymphocyte count $\left(/ \mathrm{mm}^{3}\right)[6,20]$. The GPS was defined as follows: a normal serum albumin concentration $(\geq 3.5 \mathrm{~g} / \mathrm{dL})$ and CRP level $(\leq 1.0 \mathrm{mg} / \mathrm{dL})$ was scored as 0 , a low serum albumin concentration $(<3.5 \mathrm{~g} / \mathrm{dL})$ or a high CRP level $(>1.0 \mathrm{mg} / \mathrm{dL}$ ) was scored as 1 , and a low serum albumin concentration $(<3.5 \mathrm{~g} / \mathrm{dL})$ and a high CRP level $(>1.0 \mathrm{mg} / \mathrm{dL})$ was scored as 2 [12]. The CAR was calculated by dividing the serum CRP level $(\mathrm{mg} / \mathrm{dL})$ by the serum albumin concentration $(\mathrm{g} / \mathrm{dL})$ [13]. The NLR and PLR were calculated by dividing the neutrophil or platelet count, respectively, by the lymphocyte count $[14,15]$.

\section{Follow-up}

All patients underwent routine follow-up until March 2021. Postoperative follow-up included medical history (symptoms and physical examination), laboratory tests, and imaging studies performed every 6 months for $\geq 5$ years. Patients with lymph node metastasis or 
who underwent $\mathrm{R} 1-2$ resection received postoperative adjuvant chemotherapy (tegafur/gimeracil/oteracil) for approximately 6 months.

\section{Outcomes}

The relationship between clinicopathological variables, including the PNI, and overall survival (OS) was analyzed using univariate and multivariate analyses. OS was defined as the interval between surgery and death or last follow-up. Disease-free survival (DFS) was defined as the interval between surgery and recurrence. A combined prediction model was developed using independent prognostic factors. The area under the receiver operating characteristic curve (AUC) was calculated to compare the predictive ability of each scoring system.

\section{Statistical analyses}

Data are expressed as the mean \pm standard deviation. Univariate analysis was performed using the MannWhitney $U$ test and Chi-square test. Diagnostic accuracy was determined using the AUC method. The optimal cutoff values of the PNI and other inflammation-based prognostic scores were determined by maximizing the Youden index (sensitivity+specificity-1) [21]. OS and DFS rates were estimated using the Kaplan-Meier method, and compared using the log-rank test. Univariate and multivariate analyses were performed using the Cox proportional hazards model. Prognostic factors that were statistically significant in the univariate analysis were included in the multivariate analysis. All statistical analyses were conducted using JMP (version 11; SAS Institute, Cary, NC, USA). Statistical significance was defined as $P<0.05$.

\section{Results}

\section{Patient characteristics}

Patient characteristics are summarized in Table 1 . The median age was 75 (range 38-92) years. Thirty patients had ICC; 29, GBC; 27, distal cholangiocarcinoma; 17, ampullary carcinoma; and 13, perihilar cholangiocarcinoma. Curative (R0) resection was achieved in 99 patients. Operative procedures included pancreaticoduodenectomy in 43 patients, major hepatectomy in 35, minor hepatectomy in 25 , cholecystectomy in 11 , hepatopancreaticoduodenectomy in two, and bile duct resection without hepatectomy in one. None of the patients received neoadjuvant chemotherapy or underwent preoperative portal vein embolization. Postoperative complications were observed in 44 of 116 patients: pancreatic fistula in 23 patients, bile leakage in eight, abdominal abscess in eight, pleural effusion in two, abdominal bleeding in one, and chylous ascites in one. The postoperative mortality rate was $0.0 \%$. The optimal cutoff value of the PNI was 42. Patients were stratified into a high PNI $(\geq 42)$ group $(n=88 ; 75.9 \%)$ and a low PNI $(<42)$ group $(\mathrm{n}=28 ; 24.1 \%)$, according to the cutoff value.

\section{Relationship between clinicopathological variables and the PNI}

Table 2 shows the relationship between clinicopathological variables and the PNI. Patients in the low PNI $(<42)$ group had a significantly longer mean operation time than those in the high PNI $(\geq 42)$ group (514 $\pm 220 v s$. $438 \pm 149 \mathrm{~min}$, respectively; $P=0.043)$. A significantly higher proportion of patients had lymph node metastasis in the low PNI $(<42)$ group than in the high PNI $(\geq 42)$ group.

\section{Univariate and multivariate analyses of clinicopathological factors affecting $O S$ after surgical resection}

The median OS was 43.9 (range 1-119.7) months. The 1-, 3-, and 5-year OS rates were $85.6 \%, 60.7 \%$, and $34.6 \%$, respectively. In the Kaplan-Meier analysis of all patients, the low PNI $(<42)$ group had a significantly shorter OS than the high PNI $(\geq 42)$ group $(P=0.003$; Fig. 1$)$. Table 3 shows the relationship between clinicopathological variables, including the PNI, and OS after surgical resection. In univariate analysis, OS was significantly worse in patients with lymph node metastasis $(P<0.001)$, T stage $\geq 3$ $(P<0.001)$, carbohydrate antigen $19-9$ levels $\geq 20 \mathrm{U} / \mathrm{mL}$ $(P=0.013)$, a low PLR $(<119)(P=0.003)$, preoperative cholangitis $(P=0.049)$, tumor differentiation $(P=0.003)$, an operation time $\geq 360 \mathrm{~min}(P=0.032)$, and $\mathrm{R} 1-2$ resection $(P<0.001)$. Multivariate analysis showed that a low PNI $(<42)(P=0.027)$, lymph node metastasis $(P=0.040)$, and tumor differentiation $(P=0.006)$ were significant independent predictors of OS.

\section{Univariate and multivariate analyses of clinicopathological factors affecting DFS after surgical resection}

The median DFS was 27.3 (range 1-104) months. The 1-, 3 -, and 5-year DFS rates were $57.7 \%, 44.2 \%$, and $37.5 \%$, respectively. Table 4 shows the relationship between clinicopathological variables, including the PNI, and DFS after surgical resection. In univariate analysis, DFS was significantly worse in patients with lymph node metastasis $(P<0.001)$, T stage $\geq 3(P<0.001)$, a low PNI $(<42)$ $(P=0.008)$, a high CAR $(\geq 1)(P=0.012)$, carbohydrate antigen 19-9 levels $\geq 20 \mathrm{U} / \mathrm{mL}(P=0.009)$, preoperative cholangitis $(P=0.027)$, tumor differentiation $(P=0.001)$, an operation time $\geq 360 \mathrm{~min}(P=0.006)$, and $\mathrm{R} 1-2$ resection $(P<0.001)$. Multivariate analysis showed that tumor differentiation $(P=0.016)$ was a significant independent predictor of DFS. 
Table 1 Patient characteristics

\begin{tabular}{|c|c|}
\hline Characteristic & Patients \\
\hline Age (years), mean $\pm S D$ (range) & $74.0 \pm 9.55(39-92)$ \\
\hline Sex (male/female) & $73 / 43$ \\
\hline $\mathrm{BMI}\left(\mathrm{kg} / \mathrm{m}^{2}\right)$, mean $\pm \mathrm{SD}$ (range) & $22.39 \pm 3.65(14.20-32.46)$ \\
\hline \multicolumn{2}{|l|}{ Preoperative laboratory data, mean \pm SD (range) } \\
\hline Albumin concentration (g/dL) & $3.79 \pm 0.53(1.70-4.80)$ \\
\hline Platelet count $\left(\times 10^{4} / \mathrm{mm}^{3}\right)$ & $21.65 \pm 66.61(3.37-46.30)$ \\
\hline Neutrophil count $\left(\times 10^{3} / \mathrm{mm}^{3}\right)$ & $3.75 \pm 1.88(1.01-15.39)$ \\
\hline Lymphocyte count $\left(\times 10^{3} / \mathrm{mm}^{3}\right)$ & $1.58 \pm 0.74(0.48-5.80)$ \\
\hline CRP level (mg/dL) & $1.16 \pm 2.77(0.01-24.18)$ \\
\hline CEA level (ng/mL) & $6.02 \pm 13.12(0.56-113.06)$ \\
\hline CA19-9 level (U/mL) & $1019.91 \pm 4156.75(2.00-39,284.20)$ \\
\hline PNI & $45.79 \pm 6.53(22.21-62.98)$ \\
\hline GPS (0/1/2) & $72 / 33 / 11$ \\
\hline CAR & $0.39 \pm 1.41(0.002-14.22)$ \\
\hline NLR & $2.80 \pm 2.24(0.75-14.76)$ \\
\hline PLR & $158 \pm 85(41-561)$ \\
\hline \multicolumn{2}{|l|}{ Type of cancer, n (\%) } \\
\hline Intrahepatic cholangiocarcinoma & $30(25.9)$ \\
\hline Gallbladder carcinoma & $29(25.0)$ \\
\hline Distal cholangiocarcinoma & $27(23.3)$ \\
\hline Ampullary carcinoma & $17(14.7)$ \\
\hline Perihilar cholangiocarcinoma & $13(11.2)$ \\
\hline Preoperative cholangitis, n (\%) & $43(37.1)$ \\
\hline Comorbidities (absent/present) & $34 / 82$ \\
\hline \multicolumn{2}{|l|}{ Surgical procedure, n (\%) } \\
\hline Cholecystectomy & $11(9.5)$ \\
\hline Bile duct resection w/o liver resection & $1(0.9)$ \\
\hline Minor hepatectomy & $25(21.6)$ \\
\hline Major hepatectomy & $35(30.2)$ \\
\hline Pancreaticoduodenectomy & $42(36.2)$ \\
\hline Hepatopancreaticoduodenectomy & $2(1.7)$ \\
\hline Operation time (minutes), mean $\pm \mathrm{SD}$ (range) & $457.1 \pm 171.1(124-1049)$ \\
\hline Blood loss $(\mathrm{mL})$, mean $\pm \mathrm{SD}$ (range) & $772.2 \pm 1507.8(10-13,870)$ \\
\hline Blood transfusion, n (\%) & $16(13.8)$ \\
\hline T stage $(1 / 2 / 3 / 4)$ & $18 / 39 / 51 / 8$ \\
\hline N stage (1), n (\%) & $47(40.5)$ \\
\hline UICC stage (sixth edition) (0/I/II/III/IV) & $3 / 17 / 48 / 31 / 16$ \\
\hline Resection (R0/R1/R2) & $98 / 15 / 3$ \\
\hline Tumor differentiation (well/moderate/poor/pap/well-pap/other/unknown) & $43 / 32 / 9 / 7 / 5 / 7 / 13$ \\
\hline Postoperative adjuvant chemotherapy, n (\%) & $80(69.0)$ \\
\hline Postoperative complications (Clavien-Dindo grade $\geq$ IIla) (absent/present) & $43 / 73$ \\
\hline
\end{tabular}

BMI body mass index, CA19-9 carbohydrate antigen 19-9, CAR CRP to albumin ratio, CEA carcinoembryonic antigen, CRP C-reactive protein, GPS Glasgow Prognostic Score, NLR neutrophil to lymphocyte ratio, PLR platelet to lymphocyte ratio, $P N /$ prognostic nutritional index, SD standard deviation, UICC Union for International Cancer Control, w/o without

\section{Comparison between different inflammation-based prognostic scores}

Using OS as an endpoint, the optimal cutoff values of the different inflammation-based prognostic scores were determined using the AUC method: PNI, 42 (AUC 0.613); GPS, 1 (AUC 0.580); CAR, 0.10 (AUC 0.613); NLR, 2.55 (AUC 0.520); and PLR, 120 (AUC 0.618). The AUC values of the PNI, CAR, and PLR were the highest among 
Table 2 Clinicopathological characteristics according to the PNI

\begin{tabular}{|c|c|c|c|}
\hline Characteristic & High PNI $(\geq 42)(n=88)$ & Low PNI $(<42)(n=28)$ & $P$-value \\
\hline Age (years), mean $\pm S D$ & $73.3 \pm 9.2$ & $75.6 \pm 10.9$ & 0.271 \\
\hline Sex (male/female) & $54 / 34$ & $19 / 9$ & 0.533 \\
\hline $\mathrm{BMI}\left(\mathrm{kg} / \mathrm{m}^{2}\right)$, mean $\pm \mathrm{SD}$ & $22.6 \pm 3.4$ & $21.8 \pm 4.4$ & 0.336 \\
\hline CEA level (ng/mL), mean \pm SD & $6.35 \pm 14.90$ & $4.97 \pm 4.26$ & 0.631 \\
\hline CA19-9 level (U/mL), mean \pm SD & $1072.7 \pm 4565.5$ & $852.8 \pm 2528.4$ & 0.809 \\
\hline Preoperative cholangitis (absent/present) & $58 / 30$ & $15 / 13$ & 0.243 \\
\hline Comorbidities (absent/present) & $25 / 63$ & $9 / 19$ & 0.707 \\
\hline Type of cancer (ICC/other) & $23 / 65$ & $7 / 21$ & 0.905 \\
\hline Primary disease & & & 0.130 \\
\hline ICC & 23 & 7 & \\
\hline GBC & 22 & 7 & \\
\hline Distal cholangiocarcinoma & 20 & 7 & \\
\hline Ampullary carcinoma & 16 & 1 & \\
\hline Perihilar cholangiocarcinoma & 7 & 6 & \\
\hline \multicolumn{4}{|l|}{ Surgical procedure } \\
\hline Cholecystectomy & 9 & 2 & 0.311 \\
\hline Bile duct resection w/o liver resection & 1 & 0 & \\
\hline Minor hepatectomy & 21 & 4 & \\
\hline Major hepatectomy & 22 & 13 & \\
\hline Pancreaticoduodenectomy & 34 & 8 & \\
\hline Hepatopancreaticoduodenectomy & 1 & 1 & \\
\hline Resection (R0-1/R2) & $77 / 11$ & $21 / 7$ & 0.306 \\
\hline Operation time (minutes), mean \pm SD & $438 \pm 149$ & $514 \pm 220$ & $0.043^{*}$ \\
\hline Blood loss (mL), mean \pm SD & $717 \pm 1560$ & $943 \pm 1341$ & 0.491 \\
\hline Transfusion (no/yes) & $79 / 9$ & $21 / 7$ & 0.062 \\
\hline T stage ( $\geq 3), \mathrm{n}(\%)$ & $43(48.9)$ & $16(57.1)$ & 0.445 \\
\hline N stage (1), n (\%) & $30(34.1)$ & $17(60.7)$ & $0.013^{*}$ \\
\hline UICC stage (sixth edition) (I-II/III-IV) & $56 / 32$ & $12 / 16$ & 0.053 \\
\hline Tumor differentiation (well/other) & $37 / 51$ & $11 / 17$ & 0.796 \\
\hline $\begin{array}{l}\text { Postoperative complications (Clavien-Dindo grade } \geq \text { IIla) } \\
\text { (absent/present) }\end{array}$ & $56 / 32$ & $17 / 11$ & 0.781 \\
\hline Postoperative adjuvant chemotherapy (no/yes) & $28 / 60$ & $8 / 20$ & 0.745 \\
\hline
\end{tabular}

BMI body mass index, CA19-9 carbohydrate antigen 19-9, CEA carcinoembryonic antigen, GBC gallbladder carcinoma, ICC intrahepatic cholangiocarcinoma, $P N I$ prognostic nutritional index, SD standard deviation, UICC Union for International Cancer Control, w/o without

${ }^{*} P<0.05 ;{ }^{* *} P<0.01$

the inflammation-based prognostic scores. Although the AUC value of the PNI was not the highest, the PNI was the only independent prognostic factor among the inflammation-based prognostic scores. These findings suggest that, compared to the other inflammation-based prognostic scores, the PNI is a superior prognostic factor.

\section{Combined prediction model}

A simple scoring system was developed for all patients, with 1 point assigned to each independent prognostic factor (a low PNI $[<42]$, lymph node metastasis, and tumor differentiation [poor]) using similar odds ratios as those reported in the multivariate analysis. The total score in the combined prediction model was calculated as the sum of the scores assigned to each independent prognostic factor. Accordingly, patients were divided into four groups, according to the number of risk factors $(0,1,2$, and 3 ). The proportion of patients in each group who survived was significant $(P<0.001$; Fig. $2 \mathrm{a})$. Predictive power was compared using the AUC values for each point in the scoring system (0 [AUC 0.608], 1 [AUC 0.652], 2 [AUC 0.595], and 3 [AUC 0.722]). The AUC values for 1 and 3 points in the scoring system were higher than the AUC value for the PNI alone (AUC 0.613; Fig. 2b). A combined score of the PNI and pathological findings outperformed each marker alone, in terms of discriminatory power. 
Table 3 Univariate and multivariate analyses of clinicopathological factors affecting OS after resection of BTC

\begin{tabular}{|c|c|c|c|c|}
\hline \multirow[t]{2}{*}{ Factor } & \multicolumn{2}{|c|}{ Univariate analysis } & \multicolumn{2}{|c|}{ Multivariate analysis } \\
\hline & $\mathrm{n}$ & $P$-value & $\mathrm{HR}(95 \% \mathrm{Cl})$ & $P$-value \\
\hline Age (years) & & 0.943 & - & - \\
\hline$\geq 75$ & 60 & & & \\
\hline$<75$ & 56 & & & \\
\hline Sex & & 0.090 & - & - \\
\hline Male & 73 & & & \\
\hline Female & 43 & & & \\
\hline BMI $\left(\mathrm{kg} / \mathrm{m}^{2}\right)$ & & 0.460 & - & - \\
\hline$\geq 20$ & 80 & & & \\
\hline$<20$ & 36 & & & \\
\hline CEA level (ng/mL) & & 0.847 & - & - \\
\hline$\geq 9$ & 17 & & & \\
\hline$<9$ & 99 & & & \\
\hline CA19-9 level (U/mL) & & $0.013^{*}$ & $1.12(0.57-2.23)$ & 0.733 \\
\hline$\geq 20$ & 56 & & & \\
\hline$<20$ & 60 & & & \\
\hline Preoperative cholangitis & & $0.049^{*}$ & $1.14(0.62-2.08)$ & 0.653 \\
\hline Present & 43 & & & \\
\hline Absent & 73 & & & \\
\hline Comorbidities & & 0.095 & - & - \\
\hline Present & 82 & & & \\
\hline Absent & 34 & & & \\
\hline Primary disease & & 0.090 & - & - \\
\hline ICC & 30 & & & \\
\hline $\mathrm{GBC}$ & 29 & & & \\
\hline Distal cholangiocarcinoma & 27 & & & \\
\hline Ampullary carcinoma & 17 & & & \\
\hline Perihilar cholangiocarcinoma & 13 & & & \\
\hline Surgical procedure & & 0.540 & - & - \\
\hline Cholecystectomy & 11 & & & \\
\hline Bile duct resection w/o liver resection & 1 & & & \\
\hline Minor hepatectomy & 25 & & & \\
\hline Major hepatectomy & 35 & & & \\
\hline Pancreaticoduodenectomy & 42 & & & \\
\hline Hepatopancreaticoduodenectomy & 2 & & & \\
\hline Resection & & $<0.001^{* * *}$ & $1.26(0.59-2.59)$ & 0.536 \\
\hline RO & 98 & & & \\
\hline $\mathrm{R} 1-2$ & 18 & & & \\
\hline Operation time (minutes) & & $0.032 *$ & $1.03(0.49-2.28)$ & 0.948 \\
\hline$\geq 360$ & 82 & & & \\
\hline$<360$ & 34 & & & \\
\hline Blood loss (mL) & & 0.550 & - & - \\
\hline$\geq 200$ & 81 & & & \\
\hline$<200$ & 35 & & & \\
\hline Transfusion & & 0.612 & - & - \\
\hline No & 100 & & & \\
\hline Yes & 16 & & & \\
\hline T stage & & $<0.001^{* * *}$ & $1.92(0.97-4.08)$ & 0.075 \\
\hline$<3$ & 57 & & & \\
\hline$\geq 3$ & 59 & & & \\
\hline N stage & & $<0.001^{* * *}$ & $1.99(1.03-3.90)$ & $0.040^{*}$ \\
\hline
\end{tabular}


Table 3 (continued)

\begin{tabular}{|c|c|c|c|c|}
\hline \multirow[t]{2}{*}{ Factor } & \multicolumn{2}{|c|}{ Univariate analysis } & \multicolumn{2}{|c|}{ Multivariate analysis } \\
\hline & $\mathrm{n}$ & $P$-value & $\mathrm{HR}(95 \% \mathrm{Cl})$ & $P$-value \\
\hline 0 & 69 & & & \\
\hline 1 & 47 & & & \\
\hline Tumor differentiation & & $0.003^{* *}$ & $2.45(1.29-4.83)$ & $0.006^{* *}$ \\
\hline Well & 48 & & & \\
\hline Other & 68 & & & \\
\hline PNI & & $0.003^{* *}$ & $2.12(1.09-4.04)$ & $0.027^{* *}$ \\
\hline$<42$ & 28 & & & \\
\hline$\geq 42$ & 88 & & & \\
\hline GPS & & 0.065 & - & - \\
\hline 0 & 44 & & & \\
\hline $1-2$ & 72 & & & \\
\hline CAR & & 0.064 & - & - \\
\hline$<1$ & 65 & & & \\
\hline$\geq 1$ & 51 & & & \\
\hline NLR & & 0.352 & - & - \\
\hline$<2.55$ & 85 & & & \\
\hline$\geq 2.55$ & 31 & & & \\
\hline PLR & & $0.014^{*}$ & $1.39(0.69-2.92)$ & 0.357 \\
\hline$<119$ & 68 & & & \\
\hline$\geq 119$ & 48 & & & \\
\hline Postoperative complications (Clavien-Dindo grade $\geq$ IIla) & & 0.594 & - & - \\
\hline Absent & 73 & & & \\
\hline Present & 43 & & & \\
\hline Postoperative adjuvant chemotherapy & & 0.518 & - & - \\
\hline No & 36 & & & \\
\hline Yes & 80 & & & \\
\hline
\end{tabular}

$B M I$ body mass index, $B T C$ biliary tract cancer, CA19-9 carbohydrate antigen 19-9, CAR CRP to albumin ratio, CEA carcinoembryonic antigen, $C$ confidence interval, $C R P$ C-reactive protein, GBC gallbladder carcinoma, GPS Glasgow Prognostic Score, HR hazard ratio, ICC intrahepatic cholangiocarcinoma, NLR neutrophil to lymphocyte ratio, OS overall survival, $P L R$ platelet to lymphocyte ratio, $P N /$ prognostic nutritional index, w/o without

${ }^{*} P<0.05 ;{ }^{* *} P<0.01 ;{ }^{* * *} P<0.001$

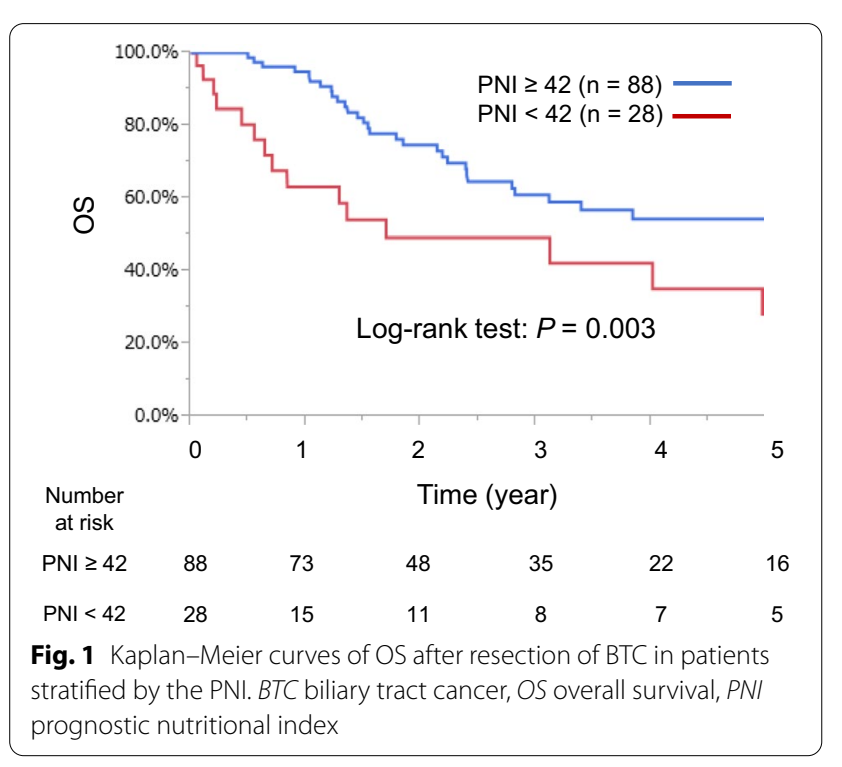

\section{Discussion}

In this study, we showed that the PNI is associated with poor prognosis after surgical resection in patients with BTC, consistent with a previous report [7]. Tumorrelated factors, including lymph node metastasis and tumor differentiation, were also found to be independent prognostic factors in multivariate analysis. Based on these findings, we developed a novel inflammationbased prognostic scoring system combining the PNI and pathological findings, which proved to be more effective than either marker alone.

A meta-analysis [22] showed that the PNI could serve as an independent prognostic factor in patients with BTC. Moreover, a high NLR and PLR may be unfavorable prognostic factors for clinical outcomes in patients with BTC [23].

The PNI, which is calculated using serum albumin concentration and total lymphocyte count, reflects the 
Table 4 Univariate and multivariate analyses of clinicopathological factors affecting DFS after resection of BTC

\begin{tabular}{|c|c|c|c|c|}
\hline \multirow[t]{2}{*}{ Factor } & \multicolumn{2}{|c|}{ Univariate analysis } & \multicolumn{2}{|c|}{ Multivariate analysis } \\
\hline & $\mathrm{n}$ & $P$-value & $\mathrm{HR}(95 \% \mathrm{Cl})$ & $P$-value \\
\hline Age (years) & & 0.316 & - & - \\
\hline$\geq 75$ & 60 & & & \\
\hline$<75$ & 56 & & & \\
\hline Sex & & 0.078 & - & - \\
\hline Male & 73 & & & \\
\hline Female & 43 & & & \\
\hline BMI $\left(\mathrm{kg} / \mathrm{m}^{2}\right)$ & & 0.294 & - & - \\
\hline$\geq 20$ & 80 & & & \\
\hline$<20$ & 36 & & & \\
\hline CEA level (ng/mL) & & 0.413 & - & - \\
\hline$\geq 9$ & 17 & & & \\
\hline$<9$ & 99 & & & \\
\hline CA19-9 level (U/mL) & & & $1.21(0.63-2.47)$ & 0.556 \\
\hline$\geq 20$ & 56 & $0.009^{* *}$ & & \\
\hline$<20$ & 60 & & & \\
\hline Preoperative cholangitis & & $0.027^{*}$ & $1.18(0.57-2.40)$ & 0.644 \\
\hline Present & 43 & & & \\
\hline Absent & 73 & & & \\
\hline Comorbidities & & 0.184 & - & - \\
\hline Present & 82 & & & \\
\hline Absent & 34 & & & \\
\hline Primary disease & & 0.060 & - & - \\
\hline ICC & 30 & & & \\
\hline $\mathrm{GBC}$ & 29 & & & \\
\hline Distal cholangiocarcinoma & 27 & & & \\
\hline Ampullary carcinoma & 17 & & & \\
\hline Perihilar cholangiocarcinoma & 13 & & & \\
\hline Surgical procedure & & 0.115 & - & - \\
\hline Cholecystectomy & 11 & & & \\
\hline Bile duct resection w/o liver resection & 1 & & & \\
\hline Minor hepatectomy & 25 & & & \\
\hline Major hepatectomy & 35 & & & \\
\hline Pancreaticoduodenectomy & 42 & & & \\
\hline Hepatopancreaticoduodenectomy & 2 & & & \\
\hline Resection & & $<0.001^{* * *}$ & $2.35(0.44-43.51)$ & \\
\hline Ro & 98 & & & 0.604 \\
\hline $\mathrm{R} 1-2$ & 18 & & & \\
\hline Operation time (min) & & $0.006^{* *}$ & $1.07(0.521-2.20)$ & 0.851 \\
\hline$\geq 360$ & 82 & & & \\
\hline$<360$ & 34 & & & \\
\hline Blood loss (mL) & & 0.478 & - & - \\
\hline$\geq 200$ & 81 & & & \\
\hline$<200$ & 35 & & & \\
\hline Transfusion & & & - & - \\
\hline No & 100 & 0.293 & & \\
\hline Yes & 16 & & & \\
\hline Tstage & & $<0.001^{* * *}$ & $1.79(0.78-4.17)$ & 0.171 \\
\hline$<3$ & 57 & & & \\
\hline
\end{tabular}


Table 4 (continued)

\begin{tabular}{|c|c|c|c|c|}
\hline \multirow[t]{2}{*}{ Factor } & \multicolumn{2}{|c|}{ Univariate analysis } & \multicolumn{2}{|c|}{ Multivariate analysis } \\
\hline & $\mathbf{n}$ & $P$-value & $\mathrm{HR}(95 \% \mathrm{Cl})$ & $P$-value \\
\hline$\geq 3$ & 59 & & & \\
\hline N stage & & $<0.001^{* * *}$ & $1.30(0.62-2.89)$ & 0.449 \\
\hline 0 & 69 & & & \\
\hline 1 & 47 & & & \\
\hline Tumor differentiation & & $0.001^{* *}$ & $2.19(1.16-4.24)$ & $0.016^{*}$ \\
\hline Well & 48 & & & \\
\hline Other & 68 & & & \\
\hline PNI & & $0.008^{* *}$ & $1.29(0.58-3.08)$ & 0.540 \\
\hline$<42$ & 28 & & & \\
\hline$\geq 42$ & 88 & & & \\
\hline GPS & & & - & - \\
\hline 0 & 44 & 0.126 & & \\
\hline $1-2$ & 72 & & & \\
\hline CAR & & $0.012^{*}$ & $1.29(0.64-2.50)$ & 0.469 \\
\hline$<1$ & 65 & & & \\
\hline$\geq 1$ & 51 & & & \\
\hline NLR & & 0.500 & - & - \\
\hline$<2.55$ & 85 & & & \\
\hline$\geq 2.55$ & 31 & & & \\
\hline PLR & & 0.269 & - & - \\
\hline$<119$ & 68 & & & \\
\hline$\geq 119$ & 48 & & & \\
\hline $\begin{array}{l}\text { Postoperative complications (Clavien-Dindo } \\
\text { grade } \geq \| l l a)\end{array}$ & & 0.449 & - & - \\
\hline Absent & 73 & & & \\
\hline Present & 43 & & & \\
\hline Postoperative adjuvant chemotherapy & & 0.222 & - & - \\
\hline No & 36 & & & \\
\hline Yes & 80 & & & \\
\hline
\end{tabular}

BMI body mass index, BTC biliary tract cancer, CA19-9 carbohydrate antigen 19-9, CEA carcinoembryonic antigen, $C l$ confidence interval, CAR CRP to albumin ratio, CRP C-reactive protein, GBC gallbladder carcinoma, GPS Glasgow Prognostic Score, HR hazard ratio, ICC intrahepatic cholangiocarcinoma, NLR neutrophil to lymphocyte ratio, $O S$ overall survival, $P L R$ platelet to lymphocyte ratio, $P N /$ prognostic nutritional index

${ }^{*} P<0.05$; ${ }^{* *} P<0.01$; ${ }^{* * *} P<0.001$

nutritional and immunological status of patients with cancer, and is a potential prognostic factor for survival. The mechanisms underlying the prognostic significance of the PNI in patients with BTC are discussed below.

Systemic inflammation has been shown to play an important role in cancer growth, invasion, and metastasis [24]. Total lymphocyte count is a component of the PNI. CD4+and CD8+ T lymphocytes are major components of the immune microenvironment [25]. Tumor-infiltrating $\mathrm{CD} 4+$ and $\mathrm{CD} 8+\mathrm{T}$ lymphocytes induce apoptosis and inhibit cancer cell proliferation [26]. Hence, lymphocytes play a critical role in cell-mediated antitumor immunity and immune surveillance [27]. Low lymphocyte counts lead to an insufficient immunological response in the tumor microenvironment, promoting cancer progression.

Malnutrition is common in patients with cancer [28], and has a negative impact on survival and recovery. Serum albumin concentration in the PNI reflects the nutritional status of patients with cancer. A low serum albumin concentration is associated with malnutrition and weight loss [29]. Hypoalbuminemia is not only a syndrome of poor nutritional status, but is also associated with a weakened host immune system [30]. Thus, a low serum albumin concentration usually predicts poor prognosis in patients with cancer. A low PNI may be predictive of an unfavorable prognosis in patients with BTC due to the aforementioned reason. 

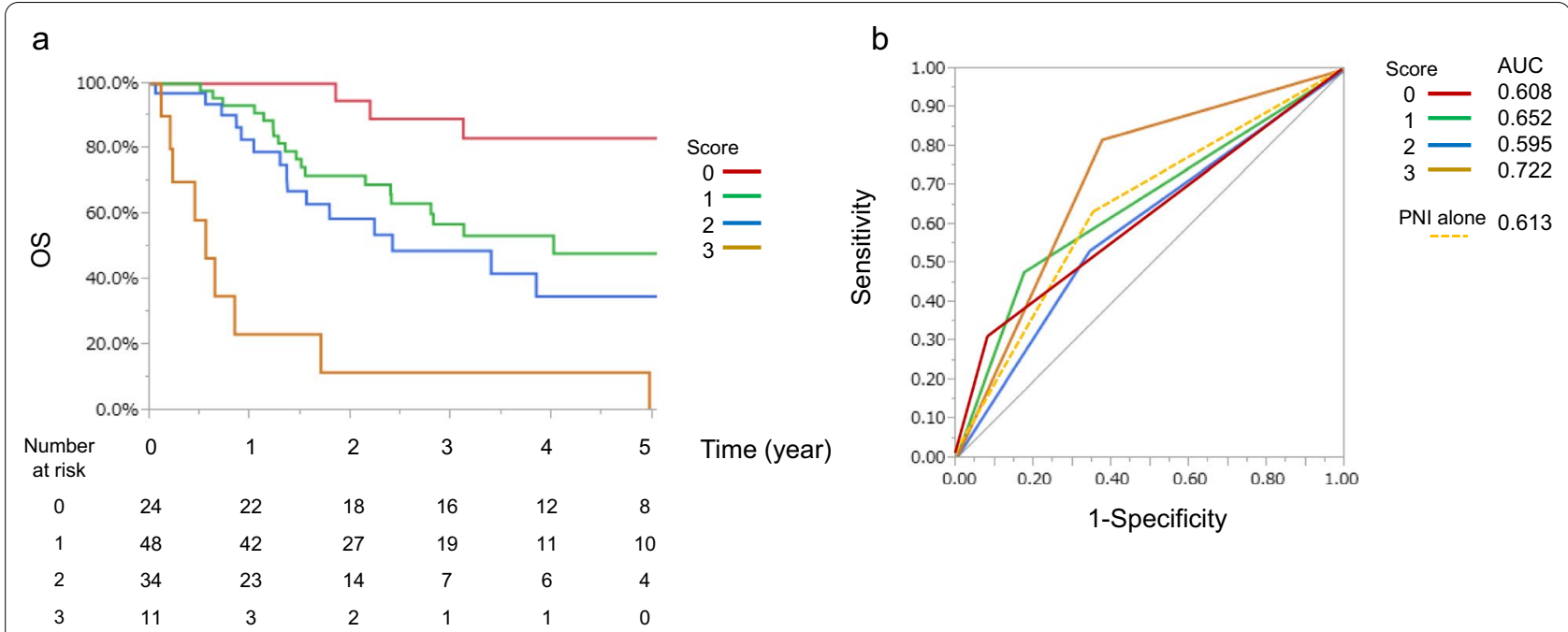

Fig. 2 Combined prediction model. a Kaplan-Meier curves of OS according to the following scoring system, with 1 point assigned to each independent prognostic factor (a low PNI [<42], lymph node metastasis, and tumor differentiation [poor]) using similar odds ratios as those reported in the multivariate analysis. The total score in the combined prediction model was calculated as the sum of the scores assigned to each independent prognostic factor. Accordingly, patients were divided into four groups, according to the number of risk factors $(0,1,2$, and 3). The proportion of patients in each group who survived was significant $(P<0.001)$. $\mathbf{b}$ Receiver operating characteristic curves of the scoring system. Predictive power was compared using the AUC for each point in the scoring system. AUC area under the curve, OS overall survival, PNI prognostic nutritional index

As discussed above, a low PNI may reflect systemic inflammation and progressive nutritional decline, resulting in poor survival. Perioperative nutritional support is recommended to improve the nutritional status of patients with hepatobiliary pancreatic carcinoma who have a high prevalence of malnutrition [31]. Preoperative immunonutrition has been reported to suppress the perioperative inflammatory response [32]. To improve prognosis, patients with a low PNI should be given immunonutrition. Further studies evaluating the relationship between immunonutrition and this inflammation-based prognostic score are required to improve the management of patients with BTC with a low PNI.

It is well known that clinicopathological characteristics, such as lymph node metastasis and tumor differentiation, significantly affect the prognosis of patients with cancer. Independent prognostic factors in this study included lymph node metastasis and tumor differentiation. Previous studies $[33,34]$ have shown that tumor differentiation is a predictor of survival after curative resection of BTC. In this study, patients with well-differentiated tumors had significantly longer survival times than those with other histologies. This was further confirmed by multivariate analysis. These findings suggest that tumor differentiation is a predictor of long-term survival. Patients with poorly differentiated tumors should be carefully monitored during postoperative follow-up to detect recurrence early.
Clinicopathological predictors have proven to be suboptimal for identifying high-risk patients. Recent evidence has underscored the discriminatory power of a combined prognostic index. Pinato et al. [35] proposed a new prognostic score for hepatocellular carcinoma, based on a combination of the modified GPS and the Cancer of the Liver Italian Program score. They reported that the predictive accuracy of the combined score was superior to that of the Cancer of the Liver Italian Program score alone. Lin et al. [36] combined the lymphocyte-to-monocyte ratio and pathological TNM stage to establish the inflammation-based pathological stage. They showed that the inflammation-based pathological stage was superior to either the pathological TNM stage or inflammation-based index alone. There are few established staging systems for BTC. In this study, we showed that the discriminatory power of a combined scoring system may be more effective than that of the PNI alone. Our combined scoring system accurately predicted prognosis, and may serve as a novel prognostic factor for patients with BTC. The combined scores reflected a poor prognosis, suggesting that more intensive follow-up or prophylactic postoperative treatment, such as chemotherapy and radiotherapy, is needed for patients with high scores.

The PNI was associated with several clinicopathological factors in this study. A low PNI was associated with lymph node metastasis and a longer operation time, 
suggesting that patients with a low PNI are at high risk of advanced disease.

This study has several limitations related to its singlecenter, retrospective design and small sample size. The sample size limited the statistical power of the multivariate and subgroup analyses. The study population was heterogeneous in terms of diagnosis and type of resection. OS rates differed for each type of BTC (ICC, GBC, extrahepatic cholangiocarcinoma, etc.), although not statistically significant. Most patients underwent radical resection. However, in patients with early-stage GBC, less invasive resections, such as cholecystectomy and liver bed resection, were more commonly performed. Future prospective, multicenter studies with larger sample sizes are needed to validate our findings.

\section{Conclusions}

A high PNI, lymph node metastasis, and tumor differentiation were independent prognostic factors for OS after surgical resection in patients with BTC. Our simple and convenient scoring system will help refine patient stratification and predict survival. In addition, a novel and powerful inflammation-based scoring system was developed. Determining indications for nutritional support with immunonutrition and more intensive follow-up or postoperative treatment, such as chemotherapy and radiotherapy, is needed for patients with a high PNI.

\begin{abstract}
Abbreviations
AUC: Area under the receiver operating characteristic curve; BTC: Biliary tract cancer; CAR: CRP to albumin ratio; CRP: C-reactive protein; DFS: Disease-free survival; GBC: Gallbladder carcinoma; GPS: Glasgow Prognostic Score; ICC: Intrahepatic cholangiocarcinoma; NLR: Neutrophil to lymphocyte ratio; OS: Overall survival; PLR: Platelet to lymphocyte ratio; PNI: Prognostic nutritional index; TNM: Tumor-node-metastasis.
\end{abstract}

\section{Acknowledgements}

We would like to thank Editage (www.editage.com) for their writing support.

\section{Authors' contributions}

$\mathrm{MU}, \mathrm{TK}, \mathrm{KK}, \mathrm{NT}, \mathrm{YY}, \mathrm{YU}, \mathrm{TN}, \mathrm{RH}, \mathrm{HM}, \mathrm{YT}$, SO, and $\mathrm{Ml}$ designed the study. MI, MU, NT, and KK treated and observed the patients. MU drafted the manuscript and performed the literature search. Ml critically revised the manuscript for important intellectual content. All authors read and approved the final manuscript.

\section{Funding}

This research did not receive any specific grant from funding agencies in the public, commercial, or not-for-profit sectors.

\section{Availability of data and materials}

The datasets used and/or analyzed during the current study are available from the corresponding author on reasonable request.

\section{Declarations}

Ethics approval and consent to participate

All procedures performed in studies involving human participants were in accordance with the ethical standards of the institutional and/or national research committee and with the 1964 Declaration of Helsinki and its later amendments or comparable ethical standards. The study design was approved by the Ethical Review Board of the National Hospital Organization Fukuyama Medical Center, Hiroshima, Japan (approval number: R2-34). The requirement for written informed consent was waived owing to the retrospective nature of the study by the Ethical Review Board of the National Hospital Organization Fukuyama Medical Center. All data were blinded before analysis.

\section{Consent for publication}

Not applicable.

\section{Competing interests}

The authors declare that they have no competing interests.

Received: 24 May 2021 Accepted: 5 October 2021

Published online: 13 October 2021

\section{References}

1. Siegel RL, Miller KD, Jemal A. Cancer statistics, 2019. CA Cancer J Clin. 2019;69:7-34.

2. Hasegawa S, Ikai I, Fujii H, Hatano E, Shimahara Y. Surgical resection of hilar cholangiocarcinoma: analysis of survival and postoperative complications. World J Surg. 2007;31:1256-63.

3. Wang Y, Li J, Xia Y, Gong R, Wang K, Yan Z, et al. Prognostic nomogram for intrahepatic cholangiocarcinoma after partial hepatectomy. J Clin Oncol. 2013;31:1188-95.

4. Buettner S, van Vugt JLA, Gaspersz MP, Coelen RJS, Roos E, Labeur TA, et al. Survival after resection of perihilar cholangiocarcinoma in patients with lymph node metastases. HPB (Oxford). 2017;19:735-40.

5. Saito H, Noji T, Okamura K, Tsuchikawa T, Shichinohe T, Hirano S. A new prognostic scoring system using factors available preoperatively to predict survival after operative resection of perihilar cholangiocarcinoma. Surgery. 2016;159:842-51.

6. Onodera T, Goseki N. Kosaki G [Prognostic nutritional index in gastrointestinal surgery of malnourished cancer patients]. Nihon Geka Gakkai Zasshi. 1984:85:1001-5.

7. Wang J, Bo X, Li M, Nan L, Wang C, Gao Z, et al. Prediction efficacy for clinical outcome of prognostic nutritional index in patients with resectable biliary tract cancer depends on sex and obstructive jaundice status. Ann Surg Oncol. 2021;28:430-8.

8. Deng Y, Pang Q, Bi JB, Zhang X, Zhang LQ, Zhou YY, et al. A promising prediction model for survival in gallbladder carcinoma patients: pretreatment prognostic nutrient index. Tumour Biol. 2016;37:15773-81.

9. Akgül Ö, Bagante F, Olsen G, Cloyd JM, Weiss M, Merath K, et al. Preoperative prognostic nutritional index predicts survival of patients with intrahepatic cholangiocarcinoma after curative resection. J Surg Oncol. 2018;118:422-30.

10. Yoo YJ, Kang CM, Choi M, Rho SY, Hwang HK, Lee WJ, et al. Preoperative prognostic nutritional index as an independent prognostic factor for resected ampulla of Vater cancer. PLOS ONE. 2020;15:e0229597.

11. Okamura Y, Ashida R, Ito T, Sugiura T, Mori K, Uesaka K. Preoperative neutrophil to lymphocyte ratio and prognostic nutritional index predict overall survival after hepatectomy for hepatocellular carcinoma. World J Surg. 2015;39:1501-9.

12. Proctor MJ, Talwar D, Balmar SM, O'Reilly DS, Foulis AK, Horgan PG, et al. The relationship between the presence and site of cancer, an inflammation-based prognostic score and biochemical parameters. Initial results of the Glasgow Inflammation Outcome Study. Br J Cancer. 2010;103:870-6.

13. Fairclough E, Cairns E, Hamilton J, Kelly C. Evaluation of a modified early warning system for acute medical admissions and comparison with C-reactive protein/albumin ratio as a predictor of patient outcome. Clin Med (Lond). 2009;9:30-3.

14. Templeton AJ, McNamara MG, Šeruga B, Vera-Badillo FE, Aneja P, Ocaña A, et al. Prognostic role of neutrophil-to-lymphocyte ratio in solid tumors: a systematic review and meta-analysis. J Natl Cancer Inst. 2014;106:124.

15. Smith RA, Bosonnet L, Raraty M, Sutton R, Neoptolemos JP, Campbell F et al. Preoperative platelet-lymphocyte ratio is an independent significant prognostic marker in resected pancreatic ductal adenocarcinoma. Am J Surg. 2009;197:466-72. 
16. Brierley J, Gospodarowicz MK, Wittekind C. TNM classification of malignant tumours. Chichester: Wiley; 2017.

17. Strasberg SM. Nomenclature of hepatic anatomy and resections: a review of the Brisbane 2000 system. J Hepatobiliary Pancreat Surg. 2005;12:351-5.

18. Couinaud C. Liver anatomy: portal (and suprahepatic) or biliary segmentation. Dig Surg. 1999;16:459-67.

19. Clavien PA, Barkun J, de Oliveira ML, Vauthey JN, Dindo D, Schulick RD, et al. The Clavien-Dindo classification of surgical complications: five-year experience. Ann Surg. 2009;250:187-96.

20. Kanda M, Fujii T, Kodera Y, Nagai S, Takeda S, Nakao A. Nutritional predictors of postoperative outcome in pancreatic cancer. Br I Surg. 2011:98:268-74.

21. Youden WJ. Index for rating diagnostic tests. Cancer. 1950;3:32-5.

22. Lv X, Zhang Z, Yuan W. Pretreatment prognostic nutritional index (PNI) as a prognostic factor in patients with biliary tract cancer: a meta-analysis. Nutr Cancer. 2020;66:1-10.

23. Spolverato G, Maqsood H, Kim Y, Margonis G, Luo T, Ejaz A, et al. Neutrophil-lymphocyte and platelet-lymphocyte ratio in patients after resection for hepato-pancreatico-biliary malignancies. J Surg Oncol. 2015;111:868-74.

24. Christofi T, Baritaki S, Falzone L, Libra M, Zaravinos A. Current perspectives in cancer immunotherapy. Cancers (Basel). 2019;11:1472.

25. Chen KJ, Zhou L, Xie HY, Ahmed TE, Feng XW, Zheng SS. Intratumoral regulatory $T$ cells alone or in combination with cytotoxic $T$ cells predict prognosis of hepatocellular carcinoma after resection. Med Oncol. 2012;29:1817-26

26. Zikos TA, Donnenberg AD, Landreneau RJ, Luketich JD, Donnenberg VS. Lung T-cell subset composition at the time of surgical resection is a prognostic indicator in non-small cell lung cancer. Cancer Immunol Immunother. 2011;60:819-27.

27. Zhou J, Ding T, Pan W, Zhu LY, Li L, Zheng L. Increased intratumoral regulatory $T$ cells are related to intratumoral macrophages and poor prognosis in hepatocellular carcinoma patients. Int J Cancer. 2009;125:1640-8.
28. Ravasco P. Nutrition in cancer patients. J Clin Med. 2019;8:1211.

29. Migita K, Takayama T, Saeki K, Matsumoto S, Wakatsuki K, Enomoto K, et al. The prognostic nutritional index predicts long-term outcomes of gastric cancer patients independent of tumor stage. Ann Surg Oncol. 2013:20:2647-54.

30. Jin Y, Zhao L, Peng F. Prognostic impact of serum albumin levels on the recurrence of stage I non-small cell lung cancer. Clinics (Sao Paulo). 2013;68:686-93.

31. Bozzetti F, Mariani L. Perioperative nutritional support of patients undergoing pancreatic surgery in the age of ERAS. Nutrition. 2014;30:1267-71.

32. Uno H, Furukawa K, Suzuki D, Shimizu H, Ohtsuka M, Kato A, et al. Immunonutrition suppresses acute inflammatory responses through modulation of resolvin E1 in patients undergoing major hepatobiliary resection. Surgery. 2016;160:228-36.

33. Hu HJ, Jin YW, Zhou RX, Ma WJ, Yang Q, Wang JK, et al. Clinical value of inflammation-based prognostic scores to predict the resectability of hyperbilirubinemia patients with potentially resectable hilar cholangiocarcinoma. J Gastrointest Surg. 2019;23:510-7.

34. Popescu I, Dumitrascu T. Curative-intent surgery for hilar cholangiocarcinoma: prognostic factors for clinical decision making. Langenbecks Arch Surg. 2014;399:693-705.

35. Pinato DJ, Stebbing J, Ishizuka M, Khan SA, Wasan HS, North BV, et al. A novel and validated prognostic index in hepatocellular carcinoma: the inflammation based index (IBI). J Hepatol. 2012;57:1013-20.

36. Lin J, Fang T, Zhu M, Xu X, Zhang J, Zheng S, et al. Comparative performance of inflammation-based prognostic scores in patients operated for intrahepatic cholangiocarcinoma. Cancer Manag Res. 2019;11:9107-19.

\section{Publisher's Note}

Springer Nature remains neutral with regard to jurisdictional claims in published maps and institutional affiliations.
Ready to submit your research? Choose BMC and benefit from:

- fast, convenient online submission

- thorough peer review by experienced researchers in your field

- rapid publication on acceptance

- support for research data, including large and complex data types

- gold Open Access which fosters wider collaboration and increased citations

- maximum visibility for your research: over $100 \mathrm{M}$ website views per year

At BMC, research is always in progress.

Learn more biomedcentral.com/submissions 